\title{
A Bound for the Least Prime Ideal in the Chebotarev Density Theorem
}

\author{
J.C. Lagarias ${ }^{1}$, H.L. Montgomery ${ }^{2}$, and A.M. Odlyzko ${ }^{3}$ \\ ${ }^{1}$ University of Maryland. College Park. MD 20742 \\ ${ }^{2}$ Universily of Michigan, Ann Arbor, MI 48104 \\ ${ }^{3}$ Bell Laboratories, Murray Hill. NJ 07974. USA
}

\section{Introduction}

We first recall the Chebotarev Density Theorem. Let $K$ be a finite algebraic extension of the rationals $\mathbf{Q}$, and $L$ a Galois extension of $K$. To each prime ideal $P$ of $K$ unramified in $L$ there corresponds a certain conjugacy class $C$ of $\operatorname{Gal}(L / K)$ consisting of the set of Frobenius automorphisms $\sigma$ attached to the prime ideals $p$ of $L$ which lie over $P$. Denote this conjugacy class by the Artin symbol $\left[\frac{L / K}{P}\right]$. For a given conjugacy class $C$ of Gal $(L / K)$ let $\pi_{C}(x)$ denote the number of prime ideals $P$ of $K$ unramified in $L$ such that $\left[\frac{L / K}{P}\right]=C$ and $N_{K / \mathbf{q}} P \leqq x$. The Chebotarev density
theorem $[6,10]$ asserts that

$$
\pi_{C}(x) \sim \frac{|C|}{|G|} \operatorname{Li}(x)
$$

as $x \rightarrow \infty$. In [7] two versions of the Chebotarev density theorem were proved, one unconditional and the other on the assumption of the Generalized Riemann Hypothesis (GRH), each of which expressed $\pi_{C}(x)$ as the sum of the main term $\frac{|C|}{|G|} L i(x)$ and an error term which is an effectively computable function of $x, \frac{|C|}{|G|}$, and the associated field constants $n_{K}=[K: \mathbf{Q}], n_{L}=[L: \mathbf{Q}]$ and $d_{K}, d_{L}$ (the absolute values of the discriminants of the two fields). Assuming the truth of the GRH for $\zeta_{L}(s)$, that paper also proved the existence of an effectively computable constant $b$ (independent of $K$ and $L$ ) such that for any conjugacy class $C$, there exists a prime ideal $P$ in $K$ with $\left[\frac{L / K}{P}\right]=C$ and

$$
N_{K / \mathbf{Q}} P \leqq h\left(\log d_{L}\right)^{2}\left(\log \log d_{L}\right)^{4} .
$$


The method of proof showed that we might specify that $N_{K / \mathbf{Q}} P$ be a rational prime. Furthermore that paper sketched a proof that sharpened this result to:

$$
N_{K / \mathbf{Q}} P \leqq b^{\prime}\left(\log d_{L}\right)^{2} .
$$

The main purpose of this paper is to supplement these results with an unconditional upper bound for the least prime ideal having a specified Artin symbol.

Theorem 1.1. There is an absolute, effectively computable constant $A_{1}$ such that for every finite extension $K$ of $\mathbf{Q}$, every finite Galois extension $L$ of $K$ and every conjugacy class $C$ of $\mathrm{Gal}(L / K)$, there exists a prime ideal $P$ of $K$ which is unramified in $L$, for which $\left[\frac{L / K}{P}\right]=C$, for which $N_{K / \mathbf{Q}} P$ is a rational prime, and which satisfies the bound

$$
N_{K / \mathbf{Q}} P \leqq 2 d_{L}^{A_{1}} .
$$

(Note. The 2 in the bound (1.3) is there only to take care of the trivial case $L=K$ $=$ Q.)

The primary technical difficulties in the proof of Theorem 1.1 center around the possible exceptional zero of $\zeta_{L}(s)$. As an auxiliary step we prove in Section 5 a version of the Deuring-Heilbronn phenomenon for $\zeta_{L}(s)$, which guarantees that, if the exceptional zero exists, then the other zeros of $\zeta_{L}(s)$ cannot lie very close to $s=1$. The proof of this auxiliary result involves the use of a power-sum inequality which is relatively easy to prove and which may be used as a substitute for Turàn's second main theorem [11] in many $L$-function applications. A feature of the proofs which distinguishes them from those of [7] is the use of kernels which weight prime ideals of small norm very heavily. These kernels enable one to obtain good lower bounds for densities of prime ideals with specified properties at the cost of not being able to estimate their number accurately. The use of different kernels is related to the "explicit formulas" of Guinand [5] and Weil [12].

The proof of Theorem 1.1 uses classical analytic methods, and does not involve any deep zero-density estimates. In the general case the bound of Theorem 1.1 is probably the best that can be obtained by current methods, since for $K=\mathbf{Q}, L$ $=\mathbf{Q}(\sqrt{D}), \rho$ the non-identity in $\operatorname{Gal}(L / K)$, the bound $(1.3)$ is equivalent to an upper bound for the least quadratic nonresidue modulo $D$, and even for this case no estimate better than (1.3) is known. However, in special cases, estimates sharper than (1.3) are possible. For example, Linnik's theorem [2] asserts the existence of an absolute effectively computable constant $B$ such that for any cyclotomic field $L$ $=\mathbf{Q}(\exp (2 \pi i / N)), N \geqq 3$, and any $\sigma \in \mathrm{Gal}(L / \mathbf{Q})$, there exists a rational prime $p$ with $\left[\frac{L / \mathbf{Q}}{(p)}\right]=\sigma$ and $p \leqq\left(\log d_{L}\right)^{B}$. Zero-density results for zeros near the line $\operatorname{Re}(s)=1$ are needed in all known proofs of Linnik's theorem. The methods of this paper do not suffice to prove Linnik's theorem, but they can be used to obtain improved bounds in some cases. For example, if $\mathrm{Gal}(L / K)$ contains a large abelian subgroup $H$, then $\zeta_{L}(s)$ can be factored into Hecke $L$-functions over the fixed field of $H$, which then leads to a better zero-free region for $\zeta_{L}(s)$, and thereby to improvements of the bound (1.3). 
The proof of Theorem 1.1 uses the known zero-free region of Hecke $L$-functions. In addition, we prove a theorem which gives a bound for the least character nonresidue of a Hecke character $\chi$ that is an explicit function of the size of a hypothetical zero-free region near $s=1$ for $\zeta_{K}(s) L(s, \chi, K)$.

Theorem 1.2. There is an absolute, effectively computable positive constant $A_{2}$ such that for every finite extension $K$ of $\mathbf{Q}$ and every Hecke character $\chi$ on $K$ the following holds: Suppose that $\zeta_{K}(s) L(s, \chi, K)$ has no zeros $\rho=\beta+i \gamma$ in the region

$$
\begin{aligned}
& 1-\delta<\beta<1, \\
& 0<|\vartheta| \leqq \delta^{2}(\log A(\chi))\left(1+\delta(\log A(\chi))^{1 / 2}\right),
\end{aligned}
$$

where

$$
\left(c_{2} \log A(\chi)\right)^{-1} \leqq \delta \leqq \frac{1}{2},
$$

and that $\zeta_{K}(s)$ has no zeros on the segment of the real axis $1-\delta \leqq s \leqq 1$. (Here $A(\chi)$ $=d_{K} N_{K / Q} f(\chi), f(\chi)$ is the conductor of $\chi$, and $c_{z}$ is a certain absolute, effectively. computable constant given by Lemma 2.3.) Then there exists a prime ideal $P$ of $K$ of degree 1 over $\mathbf{Q}$ with $\chi(P) \neq 1$ such that

$$
N_{\mathrm{K} / Q} P<\left(A_{2} \delta \log A(\chi)\right)^{\delta^{-1}} \text {. }
$$

Applying this theorem with $\delta=\frac{1}{2}$ we obtain the following corollary, which was first proved in the case $K=\mathbf{Q}$ by Ankeny [1].

Corollary 1.3. There is an absolute, effectively computable positive constant $A_{3}$ such that for every finite extension $K$ of $\mathbf{Q}$ and every Hecke character $\chi$ of $K$, if $\zeta_{K}(s) L(s, \chi, K)$ has no zeros $\rho=\beta+i \gamma$ in the region

$$
\frac{1}{2}<\beta<1 \text {, }
$$

then there exists a prime ideal $P$ of $K$ of degree 1 over $\mathbf{Q}$ with $\chi(P) \neq 1$ such that

$$
N_{K / \mathbf{Q}} P<A_{3}(\log A(\chi))^{2} .
$$

It is an open question as to what kind of zero-free region suffices to prove (1.5). Using the kernel $k(s)=x^{s} \Gamma(s)$, we can show that (1.5) holds if $\zeta_{K}(s) L(s, \chi, K)$ has no zeros in $\frac{1}{2}<\beta<1,|\gamma| \ll \log \log (A(\gamma))$. We do not know whether (1.5) could be proved if we only knew there are no zeros in $\frac{1}{2}<\beta<1,|\gamma|<\frac{1}{2}$.

Our proof of Theorem 1.1 does not suffice to show that

$$
\pi_{C}(x)>\varepsilon \frac{|C|}{|G|} \operatorname{Li}(x) \quad \text { for } x>2 d_{L}^{A_{1}},
$$

for any fixed $\varepsilon>0$. Such an estimate, or even a slightly weaker one, would be of great interest, but seems unattainable with our present techniques. However, we will sketch a proof of an unconditional upper bound estimate for $\pi_{C}(x)$ which is not difficult to prove and is of interest in some applications. 
Theorem 1.4. There exist absolute, effectively computable constants $A_{4}$ and $A_{5}$ such that if $L \neq \mathbf{Q}$ and

$$
x>\exp \left\{A_{4}\left(\log d_{L}\right)\left(\log \log d_{L}\right)\left(\log \log \log d_{L} e^{20}\right)\right\},
$$

then

$$
\pi_{C}(x) \leqq A_{5} \frac{|C|}{|G|} \operatorname{Li}(x)
$$

The paper proves Theorem 1.2 first, since it exhibits some essential features of the proof of Theorem 1.1 with less complications from the possible exceptional zero.

Throughout this paper $c_{1}, c_{2}, \ldots$ as well as all constants implied by $\ll$ and $O$ notations will denote absolute and effectively computable positive constants. Background material on properties of Hecke and Artin $L$-functions is available in [6], [7].

\section{Least Character Nonresidue}

This section presents the proof of Theorem 1.2 on the least Hecke character nonresidue. We first give an outline. A weighted sum of the values of the Hecke character at prime ideals is evaluated as an inverse Mellin transform of $-\frac{L^{\prime}}{L}(s, \chi, K)$ times a kernel function. The kernel weights only prime ideals of small norm. By contour integration this sum is equal to a sum of the kernel function over the zeros of $L(s, \chi, K)$, and this is estimated to be small. On the other hand, if $\chi(P)=1$ for all small prime ideals $P$ then the value of the Mellin transform is virtually the same as that obtained by replacing $\frac{L^{\prime}}{L}(s, \chi, K)$ with $\frac{\zeta_{K}^{\prime}}{\zeta_{K}}(s)$. This is evaluated by contour integration and is shown to be large, due to the contribution of the pole at $s=1$, provided $\zeta_{K}(s)$ has no exceptional zero. This then yields a contradiction which proves the theorem.

Proof of Theorem 1.2. We first introduce our kernel function. If $k(s)$ is an entire function, say, such that $|k(\sigma+i t)|$ is integrable as a function of $t$ for $\sigma>-\frac{1}{2}$, let

$$
\hat{k}(u)=\frac{1}{2 \pi i} \int_{a-i x}^{a+\infty} k(s) u^{-s} d s
$$

be its inverse Mellin transform, where the integration is on a vertical line $\operatorname{Re}(s)=a>-\frac{1}{2}$. The kernel function we use here is

$$
k(s)=k(s ; x, y)=\left(\frac{y^{s-1}-x^{s-1}}{s-1}\right)^{2} .
$$


A simple computation shows that for $y>x>1$ and $u>0$,

$$
\hat{k}(u ; x, y)= \begin{cases}0 & \text { if } u>y^{2}, \\ u^{-1} \log \frac{y^{2}}{u} & \text { if } x y<u<y^{2}, \\ u^{-1} \log \frac{u}{x^{2}} & \text { if } x^{2}<u<x y, \\ 0, & \text { if } u<x^{2} .\end{cases}
$$

We next come to the Hecke $L$-function. Associated to a Hecke character $\chi$ over a field $K$ is the constant

$$
A(\chi)=d_{K} N_{K / \mathbf{Q}} f(\chi)
$$

where $f(x)$ is the conductor of $\%$ We recall the Dirichlet series expansion,

$$
-\frac{L^{\prime}}{L}(s, \chi, K)=\sum_{P} \sum_{n=1}^{\infty} \chi\left(P^{n}\right) A\left(P^{n}\right)(N P)^{-n s}
$$

where $P$ runs over all prime ideals, and $A(I)$ is the generalized Von Mangoldt function

$$
A(I)= \begin{cases}\log N P & \text { if } I=P^{q} \\ 0 & \text { otherwise }\end{cases}
$$

and $N P$ denotes $N_{K / \mathbf{Q}} P$. We now consider the inverse Mellin transform (with $y>x>1)$

$$
I_{0}=\frac{1}{2 \pi i} \int_{2-i \chi}^{2+i \chi}-\frac{L}{L}(s, \chi, K) k(s ; x, y) d s=\sum_{P, n} \chi\left(P^{n}\right) A\left(P^{n}\right)(N P)^{-n} \hat{k}\left(N P^{n} ; x, y\right) .
$$

Note that the sum only counts prime-power ideals of norm $<y^{2}$. Furthermore the contribution of those ideals $P^{n}$ for which $N P^{n}$ is not a rational prime is

$$
\begin{aligned}
& \ll n_{K}(\log y) \log \left(\frac{y}{x}\right) \sum_{\substack{n=p^{a}, a \geqq 2 \\
x^{2} \leqq n \leqq r^{2}}} n^{-1} \\
& \ll n_{K}(\log y) \log \left(\frac{y}{x}\right)(x \log x)^{-1} .
\end{aligned}
$$

Next we evaluate the integral (2.5) by contour integration. Consider the integrand of (2.5) integrated on the rectangular contour with vertices at $-N+i T$, $-N-i T, 2-i T, 2+i T$. Proceeding exactly as in [7, Sect. 6], the contribution of the horizontal sides of the box goes to zero as $T \rightarrow \infty$. The contribution of the side $-N+i T,-N-i T$ goes to zero as $N \rightarrow \infty$ through values $k+\frac{1}{4}, k$ an integer. The contribution of the remaining side has as a limit the integral $I_{0}$, and by Cauchy's theorem we obtain

$$
I_{0}=-\sum_{\rho_{\chi}} k\left(\rho_{\chi} ; x, y\right)
$$

where $\rho_{\chi}$ runs over all zeros of $L(s, \chi, K)$. 
We now use (2.7) to bound the real part of (2.5) from above. First $-k(\rho ; x, y)$ is negative for real $\rho$, so we obtain a valid upper bound if we drop the terms corresponding to $\rho \in \mathbf{R}$. This takes care of the trivial zeros and any possible exceptional zero of $L(s, \chi, K)$. To proceed further we need two zero-density lemmas. The first is:

Lemma 2.1. ([7, Lemma 5.4]) Let $n_{\chi}(T)$ denote the number of zeros $\rho=\beta+i \gamma$ of the Hecke L-function $L(s, \chi, K)$ in the rectangle $0 \leqq \beta \leqq 1,|\gamma-T| \leqq 1$. Then

$$
n_{\chi}(T) \ll \log A(\chi)+n_{K} \log (|T|+2),
$$

where $A(X)=d_{K} N_{K / \mathbf{Q}} f(\chi)$.

Remark. Lemma 2.1 was proved in [7] under the tacit assumption that $\chi$ is primitive, since those were the only characters that played any role there. However, if $\chi^{\prime}$ is the primitive character that induces $\chi$, then

$$
L(s, \chi, K)=L\left(s, \chi^{\prime}, K\right) \cdot \prod_{P \mid f(\chi)}\left(1-\alpha(P)(N P)^{-s}\right),
$$

where $\alpha(P)=0$ or $\chi^{\prime}(P)$. It follows that the zeros of $L(s, \chi, K)$ are the same as those of $L\left(s, \chi^{\prime}, K\right)$, except for $\ll \log A(\chi)$ arithmetic progressions of zeros on the line $\sigma=0$, which together contain

$$
\ll(1+\Delta) \log A(\chi)
$$

zeros in any interval of length $\Delta$ on the line $\sigma=0$. Therefore the bound of the lemma above is true also for imprimitive characters. Furthermore, it is easily seen from (2.9) that

$$
\frac{L^{\prime}}{L}(s, \chi, K)=\frac{L^{\prime}}{L}\left(s, \chi^{\prime}, K\right)+O(\log A(\chi))
$$

for $\sigma=\operatorname{Re}(s) \geqq 1 / 2$, say.

The second lemma counts zeros in smaller regions. It generalizes known results for Dirichlet $L$-functions.

Lemma 2.2 Let $n(r ; s)$ denote the number of zeros $\rho$ of $L(s, \chi, K)$ with $|s-\rho| \leqq r$. Then for $\operatorname{Re}(s) \geqq 1$, and all $r>0$

$$
n(r ; s) \ll 1+r\left(\log A(\chi)+n_{K} \log (|s|+2)\right) .
$$

Proof. The estimate (2.11) is true for $r \geqq 1 / 2$ by Lemma 2.1. For the case $0<r<1 / 2$ it suffices to prove the result for $s=1+i t$, because $n(r ; 1+i t) \geqq n(r ; \sigma+i t)$ for any $\sigma \geqq 1$. We next note

$$
n(r ; 1+i t) \leqq n(2 r ;(1+r)+i t) .
$$

We now apply the estimate [7, Lemma 5.6]

$$
\left|\frac{L^{\prime}}{L}(s, \chi, K)+\frac{\delta(\chi)}{s-1}-\sum_{\substack{\rho_{\chi} \\|\gamma-t| \leqq 1}} \frac{1}{s-\rho}\right| \ll \log A(\chi)+n_{K} \log (|t|+2),
$$


valid for primitive $\chi,-\frac{1}{2} \leqq \operatorname{Re}(s) \leqq 3$, and $|s|>\frac{1}{8}$, where $\delta(\chi)=1$ if $\chi=\chi_{0}$ and 0 otherwise. By (2.10) this estimate (with a different constant implied by the $\ll$ notation) holds for any $\chi$ if $\operatorname{Re}(s) \geqq 1$. Let $s=1+r+i t$. Then

$$
\begin{aligned}
\left|\frac{L^{\prime}}{L}(s, \chi, K)\right| & \leqq\left|\frac{L^{\prime}}{L}(1+r, \chi, K)\right| \\
& \leqq\left|\frac{\zeta_{K}^{\prime}}{\zeta_{K}}(1+r, \chi, K)\right| \leqq r^{-1}+c_{1} \log d_{K},
\end{aligned}
$$

the last inequality by the equation following (8.3) in [7]. Applying this to (2.13) we have

$$
\left|\sum_{\substack{\rho_{\chi} \\|\gamma-t| \leqq 1}} \frac{1}{s-\rho}\right| \ll \log A(\chi)+n_{K} \log (|t|+2)+r^{-1} .
$$

On the other hand, if $\rho=\beta+i \gamma$,

$$
\operatorname{Re} \frac{1}{s-\rho}=\frac{(1+r)-\beta}{|s-\rho|^{2}} \geqq \frac{r}{|s-\rho|^{2}} .
$$

Consequently

$$
\left|\sum_{\substack{\rho_{\chi} \\|\gamma-t| \leqq 1}} \frac{1}{s-\rho}\right| \geqq \sum_{\substack{\rho_{\chi} \\|\gamma-t| \leqq 1}} \operatorname{Re} \frac{1}{s-\rho} \geqq n(2 r ; 1+r+i t) \cdot \frac{1}{4 r} .
$$

Substituting this result in (2.14) gives the required inequality for $n(2 r ; 1+r+i t)$, which with (2.12) completes the proof.

We also need a zero-free region for Hecke $L$-functions.

Lemma 2.3. There is an effectively computable positive absolute constant $c_{2}$ such that for all finite extensions $K$ of $\mathbf{Q}$ and Hecke characters $\chi$ on $K$, the Hecke L-function $L(s, \chi, K)$ has at most one zero $\rho=\beta+i \gamma$ with

$$
\begin{aligned}
& |\beta|>1-\left(c_{2} \log A(\chi)\right)^{-1} \\
& |\gamma|<\left(c_{2} \log A(\chi)\right)^{-1} .
\end{aligned}
$$

(If $L(s, \chi, K)=\zeta_{0}(s)$, we regard this statement as empty.) This zero can occur only if $\chi^{2}$ $=\chi_{0}$, and must be real and simple. It will be called the exceptional zero. Aside from this possible exceptional zero, $L(s, \chi, K)$ has no other zero $\rho=\beta+i \gamma$ with

$$
|\beta|>1-c_{2}^{-1}\left\{\log A(X)+n_{K} \log (|\gamma|+2)\right\}^{-1} .
$$

Proof. Note first that

$$
f(\sigma)=3+4 \cos \theta+\cos 2 \theta=2(1+\cos \theta)^{2} \geqq 0 .
$$


Let $\chi(P)=\exp \left(i \theta_{p}\right)$. Then for $\sigma>1$,

$$
\begin{array}{r}
\operatorname{Re}\left\{-3 \frac{\zeta_{K}^{\prime}}{\zeta_{K}}(\sigma)-4 \frac{L^{\prime}}{L}(\sigma+i t, \chi, K)-\frac{L^{\prime}}{L}\left(\sigma+2 i t, \chi^{2}, K\right)\right\} \\
=\sum_{P, m} f\left(m \theta_{P}-m t \log N P\right)(\log N P)(N P)^{-m \sigma} \geqq 0 .
\end{array}
$$

By (2.13) (and the remark that follows (2.13)) and the fact that $A\left(\chi^{2}\right) \leqq A(\chi)$, we find that

$$
\begin{aligned}
& -3 \frac{\zeta_{K}^{\prime}}{\zeta_{K}}(\sigma)-4 \frac{L^{\prime}}{L}(\sigma+i t, \chi, K)-\frac{L^{\prime}}{L}\left(\sigma+2 i t, \chi^{2}, K\right) \\
& =\frac{3}{\sigma-1}+\frac{4 \delta(\chi)}{\sigma-1+i t}+\frac{\delta\left(\chi^{2}\right)}{\sigma-1+2 i t}-3 \sum_{\substack{\rho_{\chi_{0}} \\
|\gamma| \leqq 1}} \frac{1}{\sigma-\rho} \\
& \quad-4 \sum_{\substack{\rho_{\chi} \\
|\gamma-t| \leqq 1}} \frac{1}{\sigma+i t-\rho}-\sum_{\substack{\rho_{\alpha^{2}} \\
|\gamma-2 t| \leqq 1}} \frac{1}{\sigma+2 i t-\rho}+O\left(\log A(\chi)+n_{K} \log (|t|+2)\right) .
\end{aligned}
$$

Now $\operatorname{Re}(s-\rho)^{-1} \geqq 0$ if $\operatorname{Re}(s) \geqq \operatorname{Re}(\rho)$. Hence if $\rho=\beta+i \gamma$ is any zero of $L(s, \chi, K)$. then the real part of the quantity in (2.17) (which is nonnegative) is

$$
\begin{aligned}
& \leqq \\
& \frac{3}{\sigma-1}+\operatorname{Re} \frac{4 \delta(\chi)}{\sigma-1+i t}+\operatorname{Re} \frac{\delta\left(\chi^{2}\right)}{\sigma-1+2 i t}-\operatorname{Re} \frac{4}{\sigma+i t-\rho} \\
& +c_{3}\left(\log A(\chi)+n_{K} \log (|t|+2)\right) .
\end{aligned}
$$

By choosing $t=\gamma$, we obtain

$$
\begin{aligned}
0 \leqq & \frac{3}{\sigma-1}-\frac{4}{\sigma-\beta}+\operatorname{Re} \frac{4 \delta(\chi)}{\sigma-1+i \gamma}+\operatorname{Re} \frac{\delta\left(\chi^{2}\right)}{\sigma-1+2 i \gamma} \\
& +c_{3}\left(\log A(\chi)+n_{K} \log (|\gamma|+2)\right) .
\end{aligned}
$$

Suppose first that $\chi^{2} \neq \chi_{0}$. Then $\delta(\chi)=\delta\left(\chi^{2}\right)=0$, and so

$$
0 \leqq \frac{3}{\sigma-1}-\frac{4}{\sigma-\beta}+c_{3}\left(\log A(\chi)+n_{K} \log (|\gamma|+2)\right) .
$$

\section{Choosing}

$$
\sigma=1+\left(100 c_{3}\right)^{-1}\left(\log A(\chi)+n_{K} \log (|\gamma|+2)\right)^{-1},
$$

say, we obtain

$$
\beta \leqq 1-\left(1000 c_{3}\right)^{-1}\left(\log A(\chi)+n_{K} \log (|\gamma|+2)\right)^{-1} .
$$

Suppose next that $\chi^{2}=\chi_{0}$, but $|\gamma| \geqq c_{3}^{-1}\left(\log A(\chi)+n_{K}\right)^{-1}$. Then (2.18) gives us

$$
0 \leqq \frac{3}{\sigma-1}-\frac{4}{\sigma-\beta}+6 c_{3}\left(\log A(\chi)+n_{K} \log (|\gamma|+2)\right),
$$

which gives (2.20) again if we choose $\sigma$ according to $(2.19)$. 
It remains to consider the case $\chi^{2}=\chi_{0}$ and $|\gamma|<c_{3}^{-1}(\log A(\chi))^{-1}$. Suppose first that $\chi \neq \chi_{0}$, but $L(s, \chi, K)$ has 2 zeros $\rho_{i}=\beta_{i}+i \gamma_{i}, 1 \leqq i \leqq 2$, with

$$
\begin{aligned}
& \left|\gamma_{i}\right|<c_{3}^{-1}\left(\log A(\chi)+n_{K}\right)^{-1}, \\
& \beta_{i}>1-\left(1000 \varepsilon_{3}\right)^{-1}\left(\log A(\chi)+n_{K}\right)^{-1} .
\end{aligned}
$$

If we now go through the derivation of (2.18) from (2.17), but keep the contributions of both $\rho_{1}$ and $\rho_{2}$, we find that for $\sigma>1, t=0$, we have

$$
0 \leqq \frac{4}{\sigma-1}-\operatorname{Re} \frac{4}{\sigma-\rho_{1}}-\operatorname{Re} \frac{4}{\sigma-\rho_{2}}+c_{3}\left(\log A(\chi)+n_{K}\right) .
$$

If we now choose

$$
\sigma=1+\left(100 c_{3}\right)^{-1}\left(\log A(\chi)+n_{K}\right)^{-1},
$$

we obtain a contradiction. Thus there cannot be 2 zeros $\rho_{i}$ as specified above. If there is a single zero satisfying (2.21) and (2.22), then it must be real, since $\chi^{2}=\chi_{0}$ means that the complex zeros of $L(s, \gamma, K)$ come in conjugate pairs.

The only remaining case is $\chi=\chi_{0}$, which is handled in an analogous way. To conclude the proof it therefore suffices to note that $n_{K} \leqq c_{4} \log A(\gamma)$ if $A(\chi) \neq 1$ (i.e., if $\left.L(s, \chi, K) \neq \zeta_{\mathbf{Q}}(s)\right)$.

To continue bounding (2.7) we divide the zeros of $L(s, \chi, K)$ off the real axis into two classes, those with $\beta \leqq 1-\delta$ and the remainder, which must have $|\gamma| \geqq \delta^{2}(\log A(\chi))\left(1+\delta(\log A(\gamma))^{1 / 2}\right)$ by hypothesis.

Consider those zeros $\rho$ with $\beta \leqq 1-\delta$. For those zeros

$$
|k(\rho ; x, y)| \leqq \frac{x^{-2 \delta}}{|\rho-1|^{2}} .
$$

Their contribution to the sum $(2,7)$ is bounded by

$$
\left.\ll x^{-2 \delta} \int_{\delta}^{\infty} \frac{1}{r^{2}} d n(r ; 1) \ll x^{-2 \delta}\left(\frac{-1}{r^{2}} n(r ; 1)\right]_{\delta}^{\infty}+\int_{\delta}^{\infty} \frac{2}{r^{3}} n(r ; 1) d r\right),
$$

which by Lemma 2.2 is

$$
\ll x^{-2 \delta}\left(\delta^{-2}+\delta^{-1} \log A(\chi)\right)
$$

since $n_{K} \ll \log |A(\gamma)|$.

Next consider those zeros $\rho$ with $\beta \geqq 1-\delta,|\gamma| \geqq \delta^{2}(\log A(\chi))\left(1+\delta(\log A(\chi))^{1 / 2}\right)$. For each such zero

$$
|k(\rho ; x, y)| \leqq(\rho-1)^{-2} .
$$

If $n^{*}(r ; 1)$ denotes the number of such zeros with $|\rho-1|<r$, then note $n^{*}(r ; 1)=0$ for

$$
r<\delta^{*}=\operatorname{Max}\left(\delta^{2}(\log A(x))\left(1+\delta(\log A(\chi))^{1 / 2}\right),\left(c_{2} \log A(\chi)\right)^{-1}\right)
$$


by Lemma 2.3 and by hypothesis. The contribution of these zeros is bounded by

$$
\left.\ll \int_{\delta^{*}}^{\infty} \frac{1}{r^{2}} d n^{*}(r, 1) \ll \frac{-1}{r^{2}} n(r ; 1)\right]_{\delta^{*}}^{\infty}+\int_{\delta^{*}}^{\infty} \frac{2}{r^{3}} n(r ; 1) d t .
$$

Using Lemma 2.2, this becomes

$$
\begin{aligned}
& \ll \delta^{*-2}+\delta^{*-1} \log A(\chi)+\delta^{*-1} n_{K}\left(\delta^{*}+2\right) \\
& \ll \delta^{*-2}+\delta^{*-1} \log \left(\delta^{*}+2\right) \log A(\chi) .
\end{aligned}
$$

In fact we will show this is

$$
\ll \delta^{-2} \text {. }
$$

To check this, note that if $\delta \geqq(\log A(\chi))^{-1}$ then $\delta^{*} \geqq \delta^{2} \log A(\chi) \geqq \delta$ while if $\delta<(\log A(\chi))^{-1}$ then $\delta^{*} \geqq\left(c_{2} \log A(\chi)\right)^{-1} \geqq c_{2}^{-1} \delta$ so that in either case $\delta^{*-2}$ $=O\left(\delta^{-2}\right)$. In order to deal with the second term, we note that $\delta^{*} \geqq \delta^{2}(\log A(\chi))(1$ $\left.+\delta(\log A(\chi))^{1 / 2}\right)$, so it will suffice to show

$$
1+\delta(\log A(\chi))^{\frac{1}{2}} \gg \log \left(\delta^{*}+2\right) .
$$

If $\delta^{*}=\left(c_{2} \log A(\chi)\right)^{-1} \leqq c_{2}^{-1}$ this is immediate, so suppose $\delta^{*}=\delta^{2}(\log A(\chi))$ (1 $\left.+\delta(\log A(\chi))^{\frac{1}{2}}\right)$. If $\delta \leqq 2(\log A(\chi))^{-\frac{1}{2}}$ then $\delta^{*} \ll 1$ and $(2.25)$ holds. If $\delta \geqq 2(\log A(\chi))^{-\frac{1}{2}}$ then $\delta^{*} \geqq 4$ and $\delta^{*} \leqq 2 \delta^{3}(\log A(\chi))^{3 / 2}$, so

$$
\log \left(\delta^{*}+2\right) \ll \log \delta^{*} \ll\left(\delta^{*}\right)^{1 / 3} \ll \delta(\log A(\chi))^{1 / 2},
$$

which again yields $(2.25)$.

Next we introduce the integral

$$
J=\frac{1}{2 \pi i} \int_{-i \infty}^{2+i \infty}\left(-\frac{\zeta_{K}^{\prime}}{\zeta}(s)\right) k(s ; x, y) d s=\sum_{P, n} A\left(N P^{n}\right)(N P)^{-n} \hat{k}\left(N P^{n} ; x, y\right) .
$$

If we now suppose that $\chi(P)=1$ for all first degree prime ideals with $N P=p<y^{2}$ for which $(P, f(\chi))=1$, then (2.26) agrees with (2.5) except possibly on those ideals $P^{n}$ for which $N P^{n} \neq p$ and also for primes $P \mid f(\chi)$. The contribution of primes dividing the conductor is

$$
\begin{aligned}
& \ll \sum_{P \mid f(x)}(\log N P) x^{-2} \log \left(\frac{y}{x}\right) \\
& \ll(\log A(\chi)) x^{-2} \log \left(\frac{y}{x}\right),
\end{aligned}
$$

and the contribution of the other primes is bounded by the quantity in (2.6), yielding

$$
\left|J-I_{0}\right| \ll n_{K} x^{-1}\left(\log \frac{y}{x}\right) \frac{\log y}{\log x}+x^{-2} \log \left(\frac{y}{x}\right) \log A(\chi) .
$$


Finally, we compute a lower bound for the integral $J$. By an argument similar to that yielding $(2.7)$ we obtain

$$
J=\left(\log \frac{y}{x}\right)^{2}-\sum_{\rho} k(\rho ; x, y)
$$

where $\rho$ runs over all the zeros of $\zeta_{K}(s)$.

We now bound the contributions of the remaining zeros, by exactly the same argument as for $L(s, \%, K)$, obtaining

$$
\left|J-\left(\log \frac{y}{x}\right)^{2}\right| \ll x^{-2 \delta} \delta^{-1} \log A(X)+\delta^{-2} .
$$

Note that the contribution of the zeros $\beta=\beta$ on the real axis with $\beta<1-\delta$ is actually included in the estimate (2.23), and there are no zeros of $\varsigma_{K}(s)$ with $1-\delta \leqq \beta<1$ by hypothesis.

We now complete the proof of Theorem 1.2 by choosing

$$
x=\left(B^{2} c_{2} \delta \log A(\chi \chi)\right)^{\frac{1}{2} \delta^{-1}}, \quad y=B^{\frac{1}{2} \delta^{-1}} x,
$$

and checking that for a sufficiently large value of $B$ (fixed once and for all) the estimates (2.25), (2.28), and (2.29) are mutually inconsistent. Recall that

$$
\left(c_{2} \log A(\chi)\right)^{-1} \leqq \delta \leqq \frac{1}{2} \text {. }
$$

We must check that

$$
\begin{aligned}
\left(\log \frac{y}{x}\right)^{2}> & c_{s}\left\{x^{-2} \delta^{-1} \log A(\chi)+\delta^{-2}+n_{K} x^{-1}\left(\log \frac{y}{x}\right)(\log y)(\log x)^{-1}\right. \\
& \left.+x^{-2} \log \frac{y}{x} \log A(\chi)\right\},
\end{aligned}
$$

where $c_{5}$ is the sum of the constants implied in (2.25), (2.28), (2.29). Now $(C \delta)^{\delta-1}$ attains its maximum as a function of $\delta$ at $e C^{-1}$. Consequently $x$ is a monotone decreasing function of $\delta$ on the interval (2.30). In particular

$$
x \geqq \frac{1}{2} B \log A(\chi)
$$

and $y \leqq x^{3 / 2}$. From (2.32) we conclude

$$
c_{5} x^{-2} \delta^{-1} \log A(\chi) \leqq 2 c_{5} \delta^{-1} \leqq c_{5}\left(\frac{1}{2} \log B\right)^{-1}\left(\log \frac{y}{x}\right)^{2}
$$

and also

$$
c_{5} n_{K} x^{-1}\left(\log \frac{y}{x}\right)\left(\frac{\log y}{\log x}\right) \ll B^{-1}\left(\log \frac{y}{x}\right)^{2}
$$

with the implied constant independent of $B$, since

$$
\frac{\log y}{\log x} \leqq \log \frac{y}{x} \quad \text { when } e^{2} \leqq x \text { and } x e^{2} \leqq y \leqq x^{3 / 2} .
$$


Next

$$
\delta^{-2} \leqq\left(\frac{1}{4} \log B\right)^{-1}\left(\log \frac{y}{x}\right)^{2}
$$

and finally

$$
x^{-2}\left(\log \frac{y}{x}\right) \log A(\chi) \leqq 4 B^{-2}(\log A(\chi))^{-1} \log \frac{y}{x} \leqq B^{-1}\left(\log \frac{y}{x}\right)^{2}
$$

if $B \geqq 100$, say. The inequalities (2.33)-(2.36) show that choosing $B$ sufficiently large will guarantee that $(2.31)$ holds. Hence there will be a prime ideal with the required properties satisfying

$$
N_{K / \mathbf{Q}} P \leqq y^{2} \leqq\left(B^{3} c_{2} \delta \log A(\chi)\right)^{\delta^{-1}}
$$

We make some remarks on proving a result similar to Theorem 1.2 for Artin $L$ functions. By a result of Stark [9, Theorem 3] we can conclude that all Artin $L$ functions $L(s, \psi, L / K)$ are analytic at any exceptional zero of $\zeta_{L}(s)$, so this causes no new difficulties. The problem is in obtaining a good upper bound for the number of singularities of $\frac{L^{\prime}}{L}(s, \psi, L / K)$ in the critical strip. Assuming Artin's conjecture on the analyticity of $L(s, \psi, L / K)$, we can get a good estimate from the functional equation, and immediately prove the analogue of Theorem 1.2, with $A(\psi)$ being the appropriate constant from the functional equation of the Artin $L$-function $L(s, \psi, L / K)$.

\section{3. $L$-Functions and Mellin Transforms}

The following sections give the proof of Theorem 1.1. As was the case with the previous proof, this proof also relies on estimates of inverse Mellin transforms

$$
\frac{1}{2 \pi i} \int_{-i \chi}^{2+i \infty} F_{C}(s) k(s) d s
$$

where $k(s)$ is a kernel function and $F_{C}(s)$ is a certain Dirichlet series which counts prime ideals whose Artin symbol is the conjugacy class $C$. We use two different kernel functions, one in the case that $\zeta_{L}(s)$ has no (exceptional) zero on the real line segment $1-\left(\log d_{L}\right)^{-2} \leqq s<1$ and the other when it does. We first show that $F_{C}(s)$, defined as a sum involving Artin $L$-functions, can be expressed as a sum involving Hecke $L$-functions, thus eliminating Artin $L$-functions from the proof. In order to get better error estimates for (3.1) if an exceptional zero of $\zeta_{L}(s)$ occurs in the range $1-\left(\log d_{L}\right)^{-1} \leqq s<1$, we show that its presence implies the existence of an enlarged zero-free region near $s=1$ (for other zeros), a result of the kind usually called the Deuring-Heilbronn phenomenon. To do this we give a simple direct proof of a powersum inequality which may be used instead of Turan's second main theorem in many $L$-function applications. Our second kernel has the property that it drops off very rapidly as we move to the left of $\sigma=1$, thus attenuating the contribution of the 
exceptional zero, at the cost of not dropping off so rapidly as we move vertically, thus necessitating a larger zero-free region to get good error bounds.

Start of Proof of Theorem 1.1. We define

$$
F_{C}(s)=-\frac{|C|}{|G|} \sum_{\psi} \bar{\psi}(g) \frac{L^{\prime}}{L}(s, \psi, L / K)
$$

where $\psi$ runs over the irreducible characters of $G$ and $L(s, \psi, L / K)$ is the Artin $L$ function attached to the character $\psi$. The orthogonality relations for the characters $\psi$ imply (see [6, Sect. 3]) that for $\operatorname{Re}(s)>1$,

$$
F_{C}(s)=\sum_{P} \sum_{m=1}^{\infty} \theta\left(P^{m}\right)(\log N P)(N P)^{-s},
$$

where for prime ideals $P$ of $K$ unramified in $L$,

$$
\theta\left(P^{m}\right)= \begin{cases}1 & \text { if }\left[\frac{L / K}{P}\right]^{m} \in C, \\ 0 & \text { otherwise, }\end{cases}
$$

and $0 \leqq \theta\left(P^{n}\right) \leqq 1$ if $P$ ramifies in $L$.

Our first reduction uses a method due to Deuring [3] to obtain an expression for $F_{c}(s)$ which involves Hecke $L$-functions rather than Artin $L$-functions, and which eliminates Artin $L$-functions from the subsequent proof. We choose $g \in C$ and let $H=\langle g\rangle$ be the cyclic group generated by $G, E$ the fixed field of $H$. Then [6, Lemma 4]

$$
F_{C}(s)=-\frac{|C|}{|G|} \sum_{\chi} \bar{\chi}(g) \frac{L^{\prime}}{L}-(s, \chi, L / E),
$$

where $\chi$ runs over the irreducible characters of $H$, which are 1-dimensional since $H$ is cyclic. By the fundamental theorem of class field theory the Artin $L$-function $L(s, \chi, L / E)$ is a certain Hecke $L$-function $L(s . \chi . E)$ attached to the field $E$, and $\chi$ is a certain primitive Hecke character, which satisfies

$$
\chi(P)=\chi\left(\left[\frac{L / E}{P}\right]\right)
$$

for all prime ideals $P$ of $E$ unramified in $L$. Hence (3.4) becomes

$$
F_{c}(s)=-\frac{|C|}{|G|} \sum_{\chi} \bar{\chi}(g) \frac{L^{\prime}}{L}(s, \chi, E)
$$

for certain Hecke $L$-functions $L(s, \not, E)$.

We now discuss the kernel functions. The two kernels we shall use are

$$
\begin{aligned}
& k_{1}(s)=k_{1}(s ; x)=k\left(s ; x, x^{2}\right)=\left(\frac{x^{2}-x^{4}}{s-1}\right)^{2} . \\
& k_{2}(s)=k_{2}(s ; x)=x^{2^{2}+} .
\end{aligned}
$$


In these kernels $x \geqq 2$ is an adjustable parameter whose value will be selected later. The dependence of the kernel on $x$ will be suppressed in what follows for notational convenience. The inverse Mellin transforms $\hat{k}_{i}(u)$ for $u>0$ of these kernels are:

$$
\begin{aligned}
& \hat{k}_{1}(u)= \begin{cases}u^{-1} \log \frac{x^{4}}{u} & x^{3} \leqq u \leqq x^{4} \\
u^{-1} \log \frac{u}{x^{2}} & x^{2} \leqq u \leqq x^{3} \\
0 & \text { otherwise }\end{cases} \\
& \hat{k}_{2}(u)=(4 \pi \log x)^{-\frac{1}{2}} \exp \left[-\frac{\left(\log \frac{u}{x}\right)^{2}}{4 \log x}\right]
\end{aligned}
$$

The sums we will estimate are

$$
I_{i}=\frac{1}{2 \pi i} \int_{2-i x}^{2+i \alpha} F_{C}(s) k_{i}(s) d s \quad(i=1,2)
$$

Using the uniform and absolute convergence of the Dirichlet series (2.4) for $\operatorname{Re}(s)>1$, and the absolute integrability of the kernels on vertical lines, we find that

$$
I_{i}=\sum_{P} \sum_{m=1}^{\infty} \theta\left(P^{m}\right)(\log N P) \hat{k}_{i}\left(N P^{m}\right),
$$

where the outer sum is over all prime ideals of $K$.

We next prove a series of three lemmas which bound the contributions to the sums in (3.9) coming from the ramified primes, the terms for which $N P^{m}$ is not prime, and (in the case of the kernel $k_{2}$ ) prime ideals with large norms. These estimates could be easily improved, but this is unnecessary for our purpose, since other error terms will be larger.

Lemma 3.1. Let $\sum^{R}$ denote summation over the prime ideals of $K$ that ramify in $L$. Then

$$
\begin{gathered}
\sum_{P}^{R} \sum_{m=1}^{\infty} \theta\left(P^{m}\right) \log (N P) \hat{k}_{1}\left(N P^{m}\right) \ll \frac{\log x}{x^{2}} \log d_{L}, \\
\sum_{P}^{R} \sum_{\substack{m=1 \\
N P^{m} \cong x^{10}}}^{\infty} \theta\left(P^{m}\right) \log (N P) \hat{k}_{2}\left(N P^{m}\right) \ll(\log x)^{\frac{1}{2}} \log d_{L} .
\end{gathered}
$$

Proof. We have

$$
\begin{aligned}
\sum_{P}^{R} \sum_{m=1}^{\infty} \theta\left(P^{m}\right) \log (N P) \hat{k}_{1}\left(N P^{m}\right) & \ll \sum_{P}{ }^{R} \log (N P) \log x \sum_{\substack{m \geqq 1 \\
N P^{m} \geqq x^{2}}}(N P)^{-m} \\
& \ll \frac{\log x}{x^{2}} \sum_{P}^{R} \log N P \ll \frac{\log x}{x^{2}} \log d_{L},
\end{aligned}
$$


since all the ramified primes divide the different $\delta(L / K)$ so the product of their norms divides $d_{L}$. Similarly

$$
\begin{aligned}
\sum_{P}^{R} \sum_{\substack{m=1 \\
N P^{m} \leqq x^{10}}}^{\infty} \theta\left(P^{m}\right) \log (N P) \hat{k}_{2}\left(N P^{m}\right) & \ll \sum_{P}^{R} \log (N P) \sum_{\substack{N^{m} \\
N x^{10}}}(\log x)^{-\frac{1}{2}} \\
& \ll(\log x)^{\frac{1}{2}} \sum_{P}^{R} \log (N P) \ll(\log x)^{\frac{1}{2}} \log d_{L} .
\end{aligned}
$$

Lemma 3.2. If $\sum^{P}$ denotes summation over those pairs $(P, m)$ for which $N P^{m}$ is not a rational prime, then

$$
\begin{aligned}
& \sum^{P} \theta\left(P^{m}\right) \log (N P) \hat{k}_{1}\left(N P^{m}\right) \ll n_{K} \frac{(\log x)^{2}}{x}, \\
& \sum^{P} \theta\left(P^{m}\right) \log (N P) \hat{k}_{2}\left(N P^{m}\right) \ll n_{K} x^{7 / 4} .
\end{aligned}
$$

Proof. If $q$ is a positive integer, there are at most $n_{K}$ distinct prime power ideals $P^{m}$ with $N P^{m}=q$. Hence

$$
\begin{aligned}
\sum_{P, m}^{P} \theta\left(P^{m}\right) \log (N P) \hat{k}_{1}\left(N P^{m}\right) \ll n_{K}(\log x)^{2} \sum_{\substack{x^{2} \leqq p^{h} \leqq x^{4} \\
p, h \geqq 2}} p^{-h} \\
\ll n_{K}(\log x)^{2} \sum_{\substack{m^{h} \geqq \frac{2}{2} \\
h \geqq 2}} m^{-h} \ll n_{K} \frac{(\log x)^{2}}{x} .
\end{aligned}
$$

For the other kernel,

$$
\begin{aligned}
\sum_{P, m}^{P} \theta\left(P^{m}\right) \log (N P) \hat{k}_{2}\left(N P^{m}\right) & \ll n_{K} \sum_{p, h \geqq 2} \log \left(p^{h}\right) \hat{k}_{2}\left(p^{h}\right) \\
& \ll n_{K} \int_{2}^{a} \log (u) \hat{k}_{2}(u) d S(u)
\end{aligned}
$$

where $S(u)$ counts the number of perfect $h$-th powers, $h \geqq 2$, which are $\leqq u$. To complete the proof we use the fact that $S(u) \ll u^{1 / 2}$ and integration by parts.

Lemma 3.3. We have

$$
\sum_{P} \sum_{\substack{m=1 \\ N P^{m} \geqq x^{10}}}^{\propto} \theta\left(P^{m}\right)(\log N P) \hat{k}_{2}\left(N P^{m}\right) \ll n_{K} x^{-10} .
$$

Proof. We have

$$
\begin{aligned}
\sum_{P} \sum_{\substack{m=1 \\
N P^{m} \geqq x^{10}}}^{\infty} \theta\left(P^{m}\right)(\log N P) \hat{k}_{2}\left(N P^{m}\right) & \ll n_{K} \sum_{q \geqq x^{10}}(\log q) \hat{k}_{2}(q) \\
& \ll n_{K} \int_{x^{10}}^{x}(\log u) \hat{k}(u) d u \ll n_{K} x^{-10} .
\end{aligned}
$$


We define

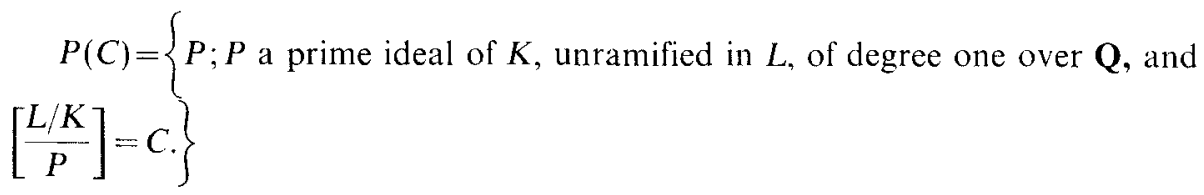

The results of the last three lemmas show that

$$
\begin{aligned}
\left|I_{1}-\sum_{P \in P(C)}(\log N P) k_{1}(N P)\right| & \ll n_{K} \frac{(\log x)^{2}}{x}+\frac{\log x}{x^{2}} \log d_{L} \\
& \ll x^{-\frac{1}{2}} \log d_{L}
\end{aligned}
$$

and

$$
\begin{aligned}
\left|I_{2}-\sum_{\substack{P \in P(C) \\
N P<x^{10}}}(\log N P) \hat{k}_{2}(N P)\right| & \ll n_{K} x^{-10}+(\log x)^{\frac{1}{2}} \log d_{L}+n_{K} x^{7 / 4} \\
& \ll x^{7 / 4} \log d_{L} .
\end{aligned}
$$

The next step of the proof consists of evaluating the integrals $I_{1}, I_{2}$ in (3.9) by contour integration. In view of the identity $(3.5)$ expressing $F_{C}(s)$ in terms of Hecke $L$-functions it suffices to evaluate integrals of the form

$$
J_{j}(\chi)=\frac{1}{2 \pi i} \int_{2-i \chi}^{2+i \chi}-\frac{L}{L}(s, \chi, E) k_{j}(s) d s,
$$

where $\chi$ is a primitive Hecke character.

In order to evaluate $J_{j}(x)$ we will consider

$$
J_{i}(\chi, T)=\frac{1}{2 \pi i} \int_{B(T)}-\frac{L^{\prime}}{L}(s, \chi, E) k_{j}(s) d s,
$$

where $B(T)$ is the positively oriented rectangle with vertices at $2-i T, 2+i T,-\frac{1}{2}$ $+i T,-\frac{1}{2}-i T$, and where $T>0$ does not equal the ordinate of any of the zeros of $L(s, \chi, E)$. By Cauchy's Theorem

$$
J_{j}(\chi, T)=\delta(\chi) k_{j}(1)-a(\chi) k_{j}(0)-\sum_{\substack{\rho \\|\gamma|<T}} k_{j}(\rho)
$$

where the last sum is over the nontrivial zeros $\rho=\beta+i \gamma$ of $L(s, \chi, E)$, counted with multiplicity, and $a(\chi)$ is the order of the zero of $L(s, \chi, E)$ at $s=0$. From the functional equation of $L(s, \chi, E)$ we deduce that $a(\chi) \leqq n_{E}$.

On the line segment from $-\frac{1}{2}+i T$ to $-\frac{1}{2}-i T$ we have [7; Lemma 6.2]

$$
\left|\frac{L^{\prime}}{L}(s, \chi, E)\right| \ll \log A(\chi)+n_{E} \log (|s|+2),
$$

so that for $j=1$ or 2 ,

$$
\left|\frac{1}{2 \pi i} \int_{-\frac{1}{2}+i T}^{-\frac{1}{2}-i T} \frac{L^{\prime}}{L}(s, \chi, E) k_{j}(s) d s\right| \ll k\left(-\frac{1}{2}\right)\left\{\log A(\chi)+n_{E}\right\}
$$


On the two segments from $2 \pm i T$ to $-\frac{1}{2} \pm i T$ we use the method of Landau [8], $[7 ;$ Sect. 6] to obtain the estimate

$$
\ll\left|k_{j}(i T)\right|\left(\log A(\chi)+n_{E} \log T\right) .
$$

If we now combine (3.18)-(3.21) and let $T \rightarrow \propto$, we obtain

$$
J_{j}(\chi)=\delta(\chi) k_{j}(1)-\sum_{\rho} k_{j}(\rho)+O\left(n_{E} k_{j}(0)\right)+O\left(k_{j}\left(-\frac{1}{2}\right)\left(\log A(x)+n_{E}\right)\right) .
$$

The definition of $F_{C}(s)$ together with (3.22) and the conductor-discriminant formula :

$$
\sum_{\chi} \log A(\chi)=\log d_{L}
$$

now show that

$$
\begin{gathered}
\frac{1}{2 \pi i} \int_{2-i \chi}^{2+i \bumpeq} F_{C}(s) k_{j}(s) d s=\frac{|C|}{|G|} k_{j}(1)-\frac{|C|}{|G|} \sum_{\chi} \bar{\chi}(g) \sum_{\rho_{\chi}} k_{j}\left(\rho_{\chi}\right) \\
+O\left(\frac{|C|}{|G|} n_{L} k_{j}(0)+\frac{|C|}{|G|} k_{j}\left(-\frac{1}{2}\right) \log d_{L}\right),
\end{gathered}
$$

where $\rho_{x}$ runs over the zeros of $L(s, \chi, E)$ in the critical strip. From (3.23) we obtain a lower bound for $I_{j}$ :

$$
\frac{|G|}{|C|} I_{j} \geqq k_{j}(1)-\sum_{\rho}\left|k_{j}(\rho)\right|-c_{6}\left[n_{L} k_{j}(0)+k_{j}\left(-\frac{1}{2}\right) \log d_{L}\right]
$$

where $\rho$ runs over the zeros of $\zeta_{L}(s)$ lying in the critical strip.

To complete the proof we need good lower bounds for the sum

$$
k_{j}(1)-\sum_{\rho}\left|k_{j}(\rho)\right| \quad(j=1,2)
$$

where $\rho$ runs over the nontrivial zeros of $\zeta_{L}(s)$. This requires additional information on the location of the zeros of $\zeta_{L}(s)$. The sum over the zeros could have a single large term coming from the exceptional zero (if it exists) and in that case we need an enlarged zero-free region to show the contribution of the other zeros is small. This enlarged region, and a lower bound on the distance of the exceptional zero from $s=1$ are the subject of the next two sections.

\section{A Power-Sum Inequality}

In this section we prove a power-sum inequality (Theorem 4.2 below) which will be used in the next section to derive an enlarged zero-free region. This result may serve as a substitute for Turàn's second main theorem [11] in many situations. 


\section{Lemma 4.1. Let}

$$
P(r, \theta)=\sum_{j=1}^{J}\left(1-\frac{j}{J+1}\right) r^{j} \cos j \theta .
$$

Then

(i) $P(r, \theta) \geqq-\frac{1}{2}$ for $0 \leqq r \leqq 1$ and all $\theta$.

(ii) $P(1,0)=\frac{J}{2}$.

(iii) $|P(r, \theta)| \leqq \frac{3}{2} r$ for $0 \leqq r \leqq \frac{1}{3}$.

Proof. $\frac{1}{2}+P(1, \theta)$ is Fejer's kernel, and hence $\geqq 0$ for all $\theta$. But if $z=r e^{i \theta}$, then $P(r, \theta)$ is a harmonic function of $z$, and hence $\geqq-\frac{1}{2}$ for $|z| \leqq 1$, proving (i). Assertion (ii) is trivial. For $0 \leqq r \leqq \frac{1}{3}$,

$$
|P(r, \theta)| \leqq \sum_{j=1}^{\infty} r^{j} \leqq \frac{3}{2} r .
$$

Theorem 4.2. Let $s_{m}=\sum_{n=1}^{\infty} b_{n} z_{n}^{m}$ and suppose that

(i) $\left|z_{n}\right| \leqq\left|z_{1}\right|$ for all $n \geqq 1$,

(ii) the $b_{n}$ are real,

(iii) $b_{n} \geqq 0$ for those $n$ for which $\frac{1}{3}\left|z_{1}\right| \leqq\left|z_{n}\right| \leqq\left|z_{1}\right|$.

Set

$$
L=\left(b_{1}\left|z_{1}\right|\right)^{-1} \sum_{n=1}^{\infty}\left|b_{n} z_{n}\right| .
$$

Then there exists $j_{0}$ with $1 \leqq j_{0} \leqq 24 L$ such that

$$
\operatorname{Re} s_{j_{0}} \geqq \frac{b_{1}}{8}\left|z_{1}\right|^{j_{0}} .
$$

Proof. By homogeneity we may suppose $\left|z_{1}\right|=1$. Let $z_{n}=r_{n} \exp \left(i \theta_{n}\right)$. In the notation of Lemma 4.1,

$$
\begin{gathered}
\sum_{j=1}^{J}\left(1-\frac{j}{J+1}\right) \operatorname{Re}\left(s_{j}\right)\left(1+\cos j \theta_{1}\right)=\sum_{n=1}^{\infty} b_{n} \sum_{j=1}^{J}\left(1-\frac{j}{J+1}\right)\left(\cos j \theta_{n}\right)\left(1+\cos j \theta_{1}\right) r_{n}^{j} \\
=\sum_{n=1}^{\infty} b_{n}\left\{P\left(r_{n}, \theta_{n}\right)+\frac{1}{2} P\left(r_{n}, \theta_{n}-\theta_{1}\right)+\frac{1}{2} P\left(r_{n}, \theta_{n}+\theta_{1}\right)\right\} .
\end{gathered}
$$

The term with $n=1$ contributes $\geqq\left(\frac{J}{4}-\frac{3}{4}\right) b_{1}>\left(\frac{J+1}{4}-3 r_{1}\right) b_{1}$. Each term with $r_{n}>\frac{1}{3}$ contributes $\geqq-b_{n} \geqq-3 r_{n} b_{n}$. Each term with $r_{n} \leqq \frac{1}{3}$ contributes $\geqq-3 r_{n}\left|b_{n}\right|$. Thus

$$
\sum_{j=1}^{J}\left(1-\frac{j}{J+1}\right)\left(\operatorname{Re} s_{j}\right)\left(1+\cos j \theta_{1}\right) \geqq \frac{J+1}{4} b_{1}-3 b_{1} L .
$$



Let $J=[24 L]$. Then the right side of $(4.1)$ is $\geqq \frac{J}{8} b_{1}$. But $\left(1-\frac{j}{J+1}\right)(1+$
$\left.\cos j \theta_{1}\right) \geqq 0$ and

$$
\sum_{j=1}^{J}\left(1-\frac{j}{J+1}\right)\left(1+\cos j \theta_{1}\right) \leqq 2 P(1,0)=J,
$$

which implies that there is at least one $j \leqq J$ such that $\operatorname{Re} s_{j} \geqq \frac{b_{1}}{8}$.

\section{The Deuring-Heilbronn Phenomenon}

The Deuring-Heilbronn phenomenon refers to the fact that if $\zeta_{L}(s)$ has a real zero $\beta_{0}$ close to $s=1$, then $\zeta_{L}(s)$ can have no other zeros nearby. The precise result is:

Theorem 5.1. There are positive, absolute, effectively computable constants $c_{7}$ and $c_{8}$ such that if $\zeta_{L}(s)$ has a real zero $\beta_{0}>0$ then $\zeta(\sigma+i t) \neq 0$ for

$$
\sigma \geqq 1-c_{8} \frac{\log \left(\frac{c_{7}}{\left(1-\beta_{0}\right) \log d_{L} \tau^{n_{L}}}\right)}{\log d_{L} \tau^{n_{L}}},
$$

where $\tau=|t|+2$, with the single exception $\sigma+i t=\beta_{0}$.

Proof. Since $(s-1) \zeta_{L}(s)$ is an entire function of order one, we have the Hadamard product

$$
(s-1) \zeta_{L}(s)=s^{r} e^{\alpha_{1}+\alpha_{2} s} \prod_{\omega}\left(1-\frac{s}{\omega}\right) e^{s / \omega},
$$

where $\omega$ runs through all the zeros of $\zeta_{L}(s), \omega \neq 0$, including the trivial ones. Differentiating (5.2) logarithmically yields

$$
-\frac{\zeta_{L}}{\zeta_{L}}(s)=\frac{1}{s-1}-\alpha_{2}-\sum_{\omega}\left(\frac{1}{s-\omega}+\frac{1}{\omega}\right)-\frac{r}{s} .
$$

The Euler product for $\zeta_{L}(s)$ gives

$$
-\frac{\zeta_{L}^{\prime}}{\zeta_{L}}(s)=\sum_{P} \sum_{m=1}^{\infty}(\log N P)(N P)^{-m s}
$$

for $\operatorname{Re} s>1$. Equating these two expansions and differentiating $2 j-1$ times, we obtain

$$
\frac{1}{(2 j-1) !} \sum_{P} \sum_{m=1}^{\infty}(\log N P)\left(\log N P^{m}\right)^{2 j-1}(N P)^{-m s}=\frac{1}{(s-1)^{2 j}}-\sum_{\omega} \frac{1}{(s-\omega)^{2 j}},
$$


where $\omega$ now runs also over the $r$ trivial zeros $\omega=0$, and this is valid for $\operatorname{Re} s>1, j \geqq 1$. Therefore if $s=\sigma+i$, we have

$$
\begin{gathered}
\frac{1}{(2 j-1) !} \sum_{P} \sum_{m=1}^{\infty}(\log N P)\left(\log N P^{m}\right)^{2 j-1} N P^{-m \sigma}\left(1+\left(N P^{m}\right)^{-i t}\right) \\
=\frac{1}{(\sigma-1)^{2 j}}+\frac{1}{(s-1)^{2 j}}-\frac{1}{\left(\sigma-\beta_{0}\right)^{2 j}}-\frac{1}{\left(s-\beta_{0}\right)^{2 j}}-\sum_{n=1}^{\infty} z_{n}^{j}
\end{gathered}
$$

where the $z_{n}$ are of the form $(\sigma-\omega)^{-2}$ or $(s-\omega)^{-2}$. The real part of the left side of (5.4) is nonnegative, so if we take $\sigma=2$, then

$$
\operatorname{Re} \sum_{n=1}^{\infty} z_{n}^{j} \leqq 1-\frac{1}{\left(2-\beta_{0}\right)^{2 j}}+\operatorname{Re}\left\{\frac{1}{(1+i t)^{2 j}}-\frac{1}{\left(2-\beta_{0}+i t\right)^{2 j}}\right\} \leqq c_{9} j\left(1-\beta_{0}\right)
$$

Suppose that $\rho=\beta+i \gamma \neq \beta_{0}$ is a zero of $\zeta_{L}(s)$. Set $t=\gamma$ in (5.5). We now apply Theorem 4.2 to the left side of (5.5). First note

$$
\left|z_{1}\right| \geqq(2-\beta)^{-2}
$$

so that

$$
\begin{aligned}
L \ll(2-\beta)^{2} \sum_{\omega}\left(\frac{1}{|2-\omega|^{2}}+\frac{1}{|2+i \gamma-\omega|^{2}}\right) & \ll \int_{0}^{\infty} \frac{1}{u^{2}+1} d n(u)+\int_{0}^{\alpha} \frac{1}{u^{2}+1} d n(u+\tau) \\
& \ll \log d_{L} \tau^{n_{L}}
\end{aligned}
$$

using the zero-density estimates for the number of zeros $n(t)$ of $\zeta_{L}(s)$ from Lemma 2.1. By Theorem 4.2 there exists some $j_{0}$ with $1 \leqq j_{0} \leqq 24 L$ such that

$$
\operatorname{Re} \sum_{n=1}^{\infty} z_{n}^{j_{0}} \geqq \frac{1}{8}(2-\beta)^{-2 j_{0}} \geqq \frac{1}{8} \exp \left(-2 j_{0}(1-\beta)\right) .
$$

Combining this with (5.5), we find that

$$
\frac{1}{8} \exp \left(-2 j_{0}(1-\beta)\right) \ll j_{0}\left(1-\beta_{0}\right) \text {. }
$$

Since $j_{0} \leqq 24 L$, which is bounded by (5.6), the desired bound follows.

From Theorem 5.1 we immediately obtain a bound on how close the exceptional zero $\beta_{0}$ can be to $s=1$.

Corollary 5.2. There is a positive, absolute, effectively computable constant $c_{10}$ such that any real zero $\beta_{0}$ of $\zeta_{L}(s)$ satisfies

$$
1-\beta_{0} \geqq d_{L}^{-c_{10}} \text {. }
$$

Proof. Choose $c_{10}$ so large that whenever $n_{L}>1$,

$$
c_{8} \log \left(\frac{c_{7} d_{L}^{c_{10}}}{\log d_{L} \cdot 2^{n_{L}}}\right)>3 \log \left(d_{L} 2^{n_{L}}\right)
$$

This is possible since $n_{L} \ll \log d_{L}$ by discriminant bounds. If we had $1-\beta_{0}$ $\left\langle d_{L}^{-c_{10}}\right.$, then by Theorem $5.1 \zeta_{L}(\sigma)$ would not vanish for $\sigma \geqq-2$, except at $\sigma$ 
$=\beta$. However $\zeta_{L}(s)$ always has a trivial zero at $s=0$ or $s=-1$, so this gives a contradiction.

Stark $[9$; Lemmas 8,11$]$ has given upper bounds for the exceptional zero that are better than the one of Corollary 5.2 in the special case that there is a sequence of fields $L_{0}=\mathbf{Q} \subseteq L_{1} \subseteq \ldots \subseteq L_{m}=L$ such that $L_{j+1} / L_{j}$ is normal.

\section{Final Estimates}

In this section we complete the proof of Theorem 1.1 by obtaining good lower bounds for the sums

$$
k_{j}(1)-\sum_{\rho}\left|k_{j}(\rho)\right| \quad(j=1,2)
$$

where $\rho$ runs over the nontrivial zeros of $\zeta_{L}(s)$.

The possible exceptional zero $\beta_{0}$ plays a special role in our estimation. To simplify the notation, we define $\beta_{0}$ to be the exceptional zero of $\zeta_{L}(s)$ if it exists, and $\beta_{0}=1-\left(c_{2} \log d_{L}\right)^{-1}$ otherwise. In either case

$$
k_{j}(1)-\sum_{\rho}\left|k_{j}(\rho)\right| \geqq k_{j}(1)-k_{j}\left(\beta_{0}\right)-\sum_{\rho \neq \beta_{0}}\left|k_{j}(\rho)\right| \quad(j=1,2) .
$$

By using the Taylor series expansions for $k_{j}(s)$ about $s=1$, we find that for $0<\beta_{0}<1$ and $x \geqq 2$,

$$
k_{1}(1)-k_{1}\left(\beta_{0}\right)=(\log x)^{2}-\left(\frac{x^{2 \beta_{0}-2}-x^{\beta_{1}-1}}{\beta_{0}-1}\right)^{2} \geqq \frac{(\log x)^{2}}{10} \operatorname{Min}\left\{1,\left(1-\beta_{0}\right) \log x\right\}
$$

and

$$
k_{2}(1)-k_{2}\left(\beta_{0}\right)=x^{2}-x^{\beta_{0}+\beta_{0}^{2}} \geqq \frac{x^{2}}{10} \operatorname{Min}\left\{1,\left(1-\beta_{0}\right) \log x\right\} .
$$

First suppose that

$$
1-\beta_{0} \geqq c_{7}^{2}\left(\log d_{L} 3^{n_{3}}\right)^{-2} \text {. }
$$

In this case we use the kernel $k_{1}(s)$. The contribution of the zeros $\rho$ of $\zeta_{L}(s)$ with $|\rho-1| \geqq 1$ is bounded by

$$
\sum_{|\rho-1| \geqq 1}\left|k_{1}(\rho)\right| \leqq \int_{i}^{\infty} \frac{2}{t^{2}} d n(t ; 1) \ll \log d_{L}
$$

by Lemma 2.2. Next suppose $|\rho-1|<1$ and $\rho=\beta+i_{i} \neq \beta_{0}$. If an exceptional zero $\beta_{0}$ exists with

$$
1-\beta_{0} \leqq \frac{1}{18} c_{2} c_{6}^{2}\left(\log d_{L}\right)^{-1}
$$

then, since $d_{L} \geqq 3^{n_{L, 1}}$ for $n_{L} \geqq 2$, we have

$$
\frac{c_{6}}{\left(1-\beta_{0}\right) \log d_{L} 3^{n_{I}}} \geqq\left\{\left(\frac{1}{2} c_{2}\right)\left(1-\beta_{0}\right) \log d_{L}\right\}^{-1 / 2}
$$


and therefore by Theorem 5.1

$$
\beta \leqq 1-c_{8} \frac{\log \left\{\frac{c_{7}}{\left(1-\beta_{0}\right) \log d_{L} 3^{n_{L}}}\right\}}{\log d_{L} 3^{n_{L}}} \leqq 1-c_{11} \frac{\log \left\{\left(\frac{1}{2} c_{2}\right)\left(1-\beta_{0}\right)\left(\log d_{L}\right)\right\}^{-1}}{\log d_{L}}
$$

On the other hand, if

$$
1-\beta_{0} \geqq \frac{1}{18} c_{2} c_{6}^{2}\left(\log d_{L}\right)^{-1},
$$

then the zero-free region of Lemma 2.3 gives

$$
\beta \leqq 1-\left(c_{2} \log d_{L}\right)^{-1} .
$$

Hence

$$
\beta \leqq 1-c_{12} \frac{\log \left\{\left(\frac{1}{2} c_{2}\right)\left(1-\beta_{0}\right)\left(\log d_{L}\right)\right\}^{-1}}{\log d_{L}}
$$

for an effectively computable positive $c_{12}$, and we may require that $c_{12}<c_{11}$. Hence (6.5) holds in all cases. Let

$$
B=c_{12} \frac{\log \left\{\left(\frac{1}{2} c_{2}\right)\left(1-\beta_{0}\right)\left(\log d_{L}\right)\right\}^{-1}}{\log d_{L}} .
$$

From (6.5) we conclude

$$
\left|k_{1}(\rho)\right| \ll x^{2(\beta-1)}|\rho-1|^{-2} \ll x^{-2 B}|\rho-1|^{-2} .
$$

By Lemma 2.2,

$$
\begin{aligned}
\sum_{\substack{|\rho-1|<1 \\
\rho \neq \beta_{0}}}\left|k_{1}(\rho)\right| \leqq x^{-2 B} \int_{B}^{1} \frac{1}{t^{2}} d n(t ; 1) & \ll x^{-2 B}\left\{B^{-2}+B^{-1} \log d_{L}\right\} \\
& \ll x^{-2 B} B^{-1} \log d_{L},
\end{aligned}
$$

since $B \gg\left(\log d_{L}\right)^{-1}$. Using (6.6) this becomes

$$
\sum_{\substack{|\rho-1|<1 \\ \rho \neq \beta_{0}}}\left|k_{1}(\rho)\right| \ll\left(\log d_{L}\right)^{2}\left\{\left(\frac{1}{2} c_{2}\right)\left(\left(1-\beta_{0}\right) \log d_{L}\right)\right\}^{2 c_{12} \frac{\log x}{\log d_{L}}}
$$

We have thus shown that

$$
\begin{aligned}
k_{1}(1) & -\sum_{\rho}\left|k_{1}(\rho)\right| \geqq \frac{1}{10} \operatorname{Min}\left\{(\log x)^{2},\left(1-\beta_{0}\right)(\log x)^{3}\right\} \\
& -c_{13} \log d_{L}-c_{14}\left(\log d_{L}\right)^{2}\left[\left(\frac{1}{2} c_{2}\right)\left(1-\beta_{0}\right) \log d_{L}\right]^{2 c_{12} \frac{\log x}{\log d_{L}}}
\end{aligned}
$$


We now complete the proof in the case $1-\beta_{0} \geqq \mathrm{c}_{7}^{2}\left(\log d_{L} 3^{n_{L}}\right)^{-2}$. Combining (6.8) with (3.15) and (3.24) we have

$$
\begin{aligned}
& \sum_{P \in P(C)}(\log N P) \hat{k}_{1}(N P) \geqq \frac{1}{10} \frac{|C|}{|G|}(\log x)^{2} \operatorname{Min}\left\{1,\left(1-\beta_{0}\right) \log x\right\} \\
& -c_{13} \frac{|C|}{|G|} \log d_{L}-c_{14} \frac{|C|}{|G|}\left(\log d_{L}\right)^{2}\left[\left(\frac{1}{2} c_{2}\right)\left(1-\beta_{0}\right) \log d_{L}\right]^{2 c_{12} \frac{\log x}{\log d_{L}}}-c_{15} x^{-\frac{1}{2}} \log d_{L} .
\end{aligned}
$$

We choose $\log x=c_{16} \log d_{L}$ with $c_{16}$ a sufficiently large absolute constant. Then the right side above is bounded below by

$$
\geqq c_{17} \frac{|C|}{|G|}\left(\log d_{L}\right)^{2} \operatorname{Min}\left\{1,\left(1-\beta_{0}\right) c_{16} \log d_{L}\right\} \geqq c_{18} \frac{|C|}{|G|}\left(\log d_{L}\right),
$$

since $1-\beta_{0} \gg\left(\log d_{L}\right)^{-2}$. In particular there is a $P \in P(C)$ with

$$
N P \leqq x^{4} \leqq d_{L}^{4 c_{16}} \text {. }
$$

Now consider the remaining case, when $1-\beta_{0} \leqq c_{7}^{2}\left(\log d_{L} 3^{n_{L}}\right)^{-2}$. In that case

$$
\log \frac{c_{7}}{\left(1-\beta_{0}\right) \log d_{L} 3^{n_{L}}} \geqq \frac{1}{2} \log \left(1-\beta_{0}\right)^{-1} .
$$

If $\rho=\beta+i \gamma$ is a zero of $\zeta_{L}(s)$ with $|y| \leqq 1$, and $\rho \neq \beta_{0}$, then by Theorem 5.1

$$
\beta \leqq 1-c_{19} \frac{\log \left(1-\beta_{0}\right)^{-1}}{\log d_{L}} .
$$

In that case

$$
\begin{aligned}
\left|k_{2}(\rho)\right| & \leqq x^{\beta^{2}+\beta} \leqq x^{1+\beta} \\
& \leqq x^{2} \exp \left[-c_{19} \frac{(\log x) \log \left(1-\beta_{0}\right)^{-1}}{\log d_{L}}\right]=x^{2}\left(1-\beta_{0}\right)^{c_{19} \frac{\log x}{\log d_{3}}} .
\end{aligned}
$$

For zeros $\rho=\beta+i \gamma$ with $|\gamma| \geqq 1$ we have

$$
\left|k_{2}(\rho)\right| \leqq x^{2-\gamma^{2}},
$$

and, by Lemma 2.1, these zeros contribute

$$
\sum_{\substack{\rho \\|y|>1}}\left|k_{2}(\rho)\right| \ll x \log d_{L} .
$$

We have shown that

$$
\begin{aligned}
k_{2}(1) & -\sum_{\rho}\left|k_{2}(\rho)\right| \geqq \frac{x^{2}}{10} \operatorname{Min}\left\{1,\left(1-\beta_{0}\right) \log x\right\} \\
& -c_{20} x \log d_{L}-c_{21} x^{2}\left(1-\beta_{0}\right)^{c_{19} \frac{\log x}{\log d_{L}}} \log d_{L} .
\end{aligned}
$$


From (3.16) and (3.24) we have

$$
\begin{aligned}
& \sum_{\substack{P \in P(C) \\
N P<x^{10}}}(\log N P) \hat{k}_{2}(N P) \geqq \frac{x^{2} \frac{|C|}{10} \operatorname{Mi} \operatorname{Min}\left\{1,\left(1-\beta_{0}\right) \log x\right\}}{} \\
& \quad-c_{20} x \log d_{L}-c_{21} x^{2}\left(1-\beta_{0}\right)^{\operatorname{cog} \frac{\log x}{\log d_{L}}} \log d_{L}-c_{22} x^{7 / 4} \log d_{L} .
\end{aligned}
$$

We now choose $x=d_{L}^{c_{23}}$ for a sufficiently large absolute constant $c_{23}$. We note that by Corollary 5.2 we have then

$$
|G| x^{7 / 4}\left(\log x^{2}\right)^{2} \log d_{L}<x^{2}\left(1-\beta_{0}\right)
$$

and hence the right side of (6.9) is

$$
\gg c_{24} \frac{|C|}{|G|} d_{L}^{2 c_{23}} \operatorname{Min}\left\{1,\left(1-\beta_{0}\right) c_{23} \log d_{L}\right\} \gg c_{24} \frac{|C|}{|G|} d_{L}^{c_{23}} .
$$

Hence there is $P \in P(C)$ with

$$
N P \leqq x^{10} \leqq d_{L}^{10_{c_{23}}} \text {. }
$$

Choosing $A_{1}=\operatorname{Max}\left(4 c_{16}, 10 c_{23}\right)$ completes the proof of Theorem 1.1.

\section{Proof of Theorem 1.4}

In this section we sketch a proof of the unconditional upper bound for the density of $P \in P(C)$. This time we use the kernel

$$
k(s)=x^{\prime} e^{s^{2}}
$$

where $x>1$, for which

$$
\hat{k}(u)=(4 \pi)^{-1 / 2} \exp \left\{-\frac{1}{4}(\log x-\log u)^{2}\right\} .
$$

Since $\hat{k}(u) \geqq 1 / 10$ for $u \in[x / 2,2 x]$, we have

$$
\begin{aligned}
I & =\frac{1}{2 \pi i} \int_{-i \infty}^{2+i \infty} F_{C}(s) k(s) d s=\sum_{P} \sum_{m=1}^{\infty} \theta\left(P^{m}\right)(\log N P) \cdot \hat{k}\left(N P^{m}\right) \\
& \geqq \frac{\log x}{20} \cdot\left\{\pi_{C}(x)-\pi_{C}(x / 2)\right\} .
\end{aligned}
$$

On the other hand, by shifting the line of integration to $\operatorname{Re}(s)=-1 / 4$, we find that

$$
I=\frac{|C|}{|G|} x e-\frac{|C|}{|G|} \sum_{\chi} \bar{\chi}(g) \sum_{\rho_{\chi}} x^{\rho} e^{\rho^{2}}+O\left(\frac{|C|}{|G|} x^{-1 / 4} \log d_{L}\right)+O\left(\frac{|C|}{|G|} n_{L}\right),
$$

where $\rho=\rho_{\chi}$ runs over the nontrivial zeros of $L(s, \chi, E)$, the last term comes from the singularity at $s=0$, and the penultimate summand is due to the integral along 
$\operatorname{Re}(s)=-1 / 4$. We wish to prove that $|I| \ll|C| x /|G|$. Now if $x \geqq d_{L}$, then

$$
\begin{aligned}
& n_{L} \ll \log d_{L} \ll x \\
& x^{-1 / 4} \log d_{L} \ll 1 .
\end{aligned}
$$

The exceptional zero (if it exists) gives

$$
\left|x^{\rho} e^{\rho^{2}}\right| \leqq x e \ll x .
$$

By Lemma 2.3 any other zero $\rho=\beta+i \gamma$ satisfies

$$
\beta \leqq 1-\left(c_{2} \log d_{L}(|\gamma|+2)^{n_{L}}\right)^{-1},
$$

and so if $|i|<\log \log d_{L}$, then

$$
\beta \leqq 1-c_{25}\left(\log d_{L}\right)^{-1}\left(\log \log \log d_{L} e^{20}\right)^{-1} .
$$

Therefore

$$
\begin{aligned}
\sum_{\rho}\left|x^{\rho} e^{\rho^{2}}\right| \ll & +x \sum_{|,| \geq \log \log d_{L}} e^{-r^{2}} \\
& +x \exp \left\{-\frac{c_{25} \log x}{\log d_{L} \log \log \log d_{L} e^{20}}\right\} \sum_{|,|<\mid<\log \log d_{t}} e^{-r^{2}} \\
\ll & +x\left(\log d_{L}\right)^{-1}+x\left(\log d_{L}\right) \exp \left\{-\frac{c_{25} \log x}{\log d_{L} \log \log \log d_{L} e^{20}}\right\} .
\end{aligned}
$$

If

$$
\log x \geqq c_{26}\left(\log d_{L}\right)\left(\log \log d_{L}\right)\left(\log \log \log d_{L} e^{20}\right)
$$

then the right side of $(7.5)$ is $\ll x$. Combining this with (7.1)-(7.4), we find that

$$
\pi_{C}(2 x)-\pi_{C}(x) \ll \frac{|C|}{|G|} \frac{x}{\log x}
$$

if (7.6) is satisfied. Now for any $x \geqq 2$,

$$
\pi_{C}(x) \leqq n_{L} \pi(x) \ll n_{L} \frac{x}{\log x} .
$$

Therefore if $A_{4}=10 c_{26}$, and

$x \log x \geqq A_{4}\left(\log d_{L}\right)\left(\log \log d_{L}\right)\left(\log \log \log d_{L} e^{20}\right)$,

then (7.7) and (7.8) yield the assertion of Theorem 1.4.

\section{References}

1. Ankeny. N.C.: The least quadratic non-residue. Annals of Math. 55. 65-72(1952)

2. Bombieri, E.: Le grand crible dans la théorie analytique des nombres. Astérisque No. 18 , Société Mathématique de France, Paris 1974 
3. Deuring, M.: Über den Tschebotareffschen Dichtigkeitssatz. Math. Annalen 110, 414-415 (1934)

4. Fogels, E.: On the distribution of prime ideals. Acta Arithmetica 7, 255-269 (1961/62)

5. Guinand, A.P.: A summation formula in the theory of prime numbers. Proc. London Math. Soc. (2) 50, 107-119 (1948)

6. Heilbronn, H.: Zeta functions and $L$-functions. In: Algebraic Number Theory (J.W.S. Cassels and A. Fröhlich, eds.) pp. 204-230. New York, London: Academic Press, 1967

7. Lagarias, J.C.: Odlyzko, A.M.: Effective Versions of the Chebotarev Density Theorem. In: Algebraic Number Fields, L-Functions and Galois Properties (A. Fröhlich, ed.), pp. 409-464. New York, London: Academic Press 1977

8. Landau, E.: Algebraische Zahlen, Göttingen 1927

9. Stark, H.M.: Some effective cases of the Brauer-Siegel theorem. Inventiones math. 23, 135-152 (1974)

10. Tschebotareff, N.: Die Bestimmung der Dichtigkeit einer Menge von Primzahlen welche zu einer gegebenen Substitutionenklasse gehören. Math. Annalen 95, 191-228 (1926)

11. Turàn, P.: Eine neue Methode in der Analysis und deren Anwendungen. Budapest: Akademiai Kiadó 1953

12. Weil, A.: Sur les "formules explicites" de la théorie des nombres premiers. Comm. Sem. Math. Lund 252-265 (1952)

Received June 5, 1979 